\title{
Reconstructing permutations from identification minors
}

\author{
Erkko Lehtonen \\ CEMAT-CIÊNCIAS, Departamento de Matemática, Faculdade de Ciências \\ Universidade de Lisboa, 1749-016 Lisboa, Portugal \\ Technische Universität Dresden, Institut für Algebra \\ 01062 Dresden, Germany \\ Erkko.Lehtonen@tu-dresden.de
}

Submitted: Jun 23, 2015; Accepted: Oct 15, 2015; Published: Oct 30, 2015

Mathematics Subject Classifications: 05A05

\begin{abstract}
We consider the problem whether a permutation of a finite set is uniquely determined by its identification minors. While there exist non-reconstructible permutations of every set with two, three, or four elements, we show that every permutation of a finite set with at least five elements is reconstructible from its identification minors. Moreover, we provide an algorithm for recovering a permutation from its deck. We also discuss a generalization of this reconstruction problem, as well as the related set-reconstruction problem.
\end{abstract}

Keywords: reconstruction problem, permutation, identification minor

\section{Introduction}

Reconstruction problems are a general class of mathematical problems that concern whether a mathematical object is uniquely determined by pieces of partial information about the object. In this paper, we will discuss reconstruction problems that fall into a general framework that was elegantly formalized by Couceiro, Schölzel, and the current author in [4, Section 2.2]. Roughly speaking, a reconstruction problem comprises a set $\mathcal{O}$ of objects, a way of forming from each object $O \in \mathcal{O}$ certain derived objects called the cards of $O$, and an equivalence relation on $\mathcal{O}$. Once this data is specified, we may ask whether, or to what extent, an object is uniquely determined, up to equivalence, by its deck, i.e., the collection of (the equivalence classes of) its cards.

Note that a given object may give rise to the same derived object, up to equivalence, in many different ways, and it is important to keep track of the number of times each card appears in the deck. In other words, by a "collection of cards" we mean a multiset 
of cards. Ignoring the multiplicities, taking "collection" to mean a set, we are dealing with a somewhat different yet related problem, often referred to as a set-reconstruction problem.

Perhaps one of the most renowned reconstruction problem comes from graph theory. Is every graph with at least three vertices uniquely determined, up to isomorphism, by the collection of its one-vertex-deleted subgraphs? It was conjectured by Kelly [7] and Ulam [15] that the answer to this question is positive, but this still remains as an open problem that has received considerable attention over the years.

Analogous reconstruction problems have been formulated for many other types of mathematical objects, such as directed graphs, hypergraphs, relations, posets, functions, matrices, matroids, integer partitions, and so on. The main topic of this paper is reconstruction problems the objects of which are permutations.

Evidently, there are many possibilities for defining how the cards of a permutation are formed, and various reconstruction problems involving permutations have accordingly been formalized in the literature. For instance, Ginsburg [6], Raykova [13], and Smith [14] have studied the reconstruction problem where, for a fixed parameter $k \in \mathbb{N}_{+}$, the cards of a permutation $\pi=\pi_{1} \pi_{2} \ldots \pi_{n}$ are the so-called $(n-k)$-minors of $\pi$, i.e., the patterns of the subsequences of $\pi$ of length $n-k$. For example, the patterns of the subsequences of length 3 of the permutation 31524 are 213, 312, 213, 231, 132, 213, 132, 132, 123, 312 .

Monks [11] considered the variant in which the cards of a permutation $\pi$ are the socalled cycle minors of $\pi$. The cycle minors of a permutation $\pi \in \Sigma_{n}$ are formed by first deleting an entry $i \in[n]$ from the representation of $\pi$ as a product of disjoint cycles, and then subtracting 1 from every number greater than $i$. For example, the cycle minors of $(134)(25)$ are (23)(14), (13)(24), (13)(24), (123)(4), (134)(2).

In this paper, we study yet another reconstruction problem of permutations. In this variant, the cards of a permutation are its identification minors. The identification minors of $\pi=\pi_{1} \pi_{2} \ldots \pi_{n}$ are formed, for each two-element subset $\{i, j\}(i<j)$ of $\{1, \ldots, n\}$, by replacing the entry $j$ by $i$, deleting the second occurrence of $i$, and taking the pattern of the resulting sequence. For example, the identification minors of 31524 are 1423, 3124, $2143,3142,3124,2143,3142,3124,3142,3142$.

The identification minors of permutations are in a complete analogy to the identification minors of functions of several arguments. The formation of minors is a way of deriving new functions from a given one that has great significance in universal algebra. A function $f: A^{n} \rightarrow B$ is said to be a minor of another function $g: A^{m} \rightarrow B$, if $f$ can be obtained from $g$ through a combination of the following operations: introduction of inessential arguments, deletion of inessential arguments, identification of arguments, and permutation of arguments. In the special case when $f$ is obtained from $g$ through the identification of a single pair of arguments, $f$ is called an identification minor of $g$. Examples of recent work on minors of functions include the papers by Couceiro and Foldes [1], Couceiro, Schölzel, and the current author [2], Ekin, Foldes, Hammer, and Hellerstein [5], Pippenger [12], Wang [16], Willard [17], and Zverovich [18].

A reconstruction problem for functions and identification minors was formulated in [8]: the objects are functions $A^{n} \rightarrow B$, the cards of a function $f: A^{n} \rightarrow B$ are its identification 
minors, and two $n$-ary functions are considered equivalent if each one can be obtained from the other through permutation of arguments. Several results, both positive and negative, concerning this reconstruction problem were obtained by the current author, partly in collaboration with Couceiro and Schölzel in [3, 4, 8, 9, 10].

Certain quotient-like structures of permutations, called "permutations arising from a permutation via partitions", arose as a crucial tool in [10], where the current author studied the reconstructibility of functions determined by the order of first occurrence. (We will discuss this in a bit more detail in Section 6.) The identification minors of permutations, as defined above, constitute an important special case of permutations arising from a permutation via partitions. Against this background, it looks very natural to investigate the reconstruction problem of permutations and identification minors that is the topic of this paper.

This paper is organized as follows. In Section 2, we provide the necessary definitions and we make the immediate observation that if $2 \leqslant n \leqslant 4$, then there are permutations of $\{1, \ldots, n\}$ that are not reconstructible from identification minor. In fact, if $n=2$ or $n=3$, then every permutation of $\{1, \ldots, n\}$ is non-reconstructible. In Section 3, we then prove the main result of this paper: for $n \geqslant 5$, every permutation of $\{1, \ldots, n\}$ is reconstructible from its identification minors (Theorem 3.7). In fact, the proof provides an effective algorithm for actually recovering a permutation from its deck; this is explained in Section 4. In Section 5, we discuss the related set-reconstruction problem and observe that not every permutation of $\{1, \ldots, n\}$ is set-reconstructible, for all $n \geqslant 2$, i.e., if the multiplicities of cards are ignored, then reconstruction is no longer possible in general, no matter how large $n$ is. Finally, in Section 6, we make some conclusive remarks and suggest a natural generalization of the reconstruction problem discussed in this paper. Analysis of this generalization remains a topic of further research.

\section{Identification minors of permutations}

\subsection{Notation}

We will first introduce some general notation which we will use throughout the paper. The set of positive integers is denoted by $\mathbb{N}_{+}$. For $n \in \mathbb{N}_{+}$, the set $\{1, \ldots, n\}$ is denoted by $[n]$. The symmetric group of $[n]$ is denoted by $\Sigma_{n}$. Any permutation $\pi \in \Sigma_{n}$ corresponds to the sequence (or word) $\pi_{1} \pi_{2} \ldots \pi_{n}$, where $\pi_{i}=\pi(i)$ for every $i \in[n]$. If $\pi(i)=j$, then we say that $j$ is the location of $i$ in $\pi$, or that $i$ is at the $j$-th position in $\pi$. We write $i<_{\pi} j$ to denote the fact that $i$ is located to the left of $j$ in the sequence $\pi_{1} \pi_{2} \ldots \pi_{n}$, i.e., $\pi^{-1}(i)<\pi^{-1}(j)$. The pattern of a sequence $a_{1} a_{2} \ldots a_{\ell}$ of integers with no repeated entries is the unique permutation $\pi=\pi_{1} \pi_{2} \ldots \pi_{\ell} \in \Sigma_{\ell}$ such that $a_{1} a_{2} \ldots a_{\ell}$ is order-isomorphic to $\pi_{1} \pi_{2} \ldots \pi_{\ell}$, i.e., the relative order of elements is the same in both sequences.

The set of all 2-element subsets of $[n]$ is denoted by $\left(\begin{array}{c}{[n]} \\ 2\end{array}\right)$. For a permutation $\pi \in \Sigma_{n}$ and a 2 -element set $I=\{i, j\} \in\left(\begin{array}{c}{[n]} \\ 2\end{array}\right)$ with $i<j$, let $\pi^{I}$ be the permutation of $[n-1]$ that is obtained from the sequence $\pi_{1} \pi_{2} \ldots \pi_{n}$ by performing the following steps:

1. Replace $j$ by $i$ and delete the rightmost occurrence of $i$ in the resulting sequence. 
(Equivalently, if $j<_{\pi} i$ then swap $i$ and $j$. Delete $j$ from the resulting sequence.)

2. Decrease any number greater than $j$ in the resulting sequence by 1. (Equivalently, take the pattern of the resulting sequence.)

The permutations of the form $\pi^{I}$ for some $I \in\left(\begin{array}{c}{[n]} \\ 2\end{array}\right)$ are called identification minors of $\pi$.

\subsection{Reconstruction problem}

Now we can formulate a reconstruction problem for permutations and identification minors. The objects are permutations $\pi \in \Sigma_{n}$ for some $n \in \mathbb{N}_{+}$. The cards of $\pi \in \Sigma_{n}$ are the $\left(\begin{array}{l}n \\ 2\end{array}\right)$ identification minors $\pi^{I}$ of $\pi\left(I \in\left(\begin{array}{c}{[n]} \\ 2\end{array}\right)\right)$. The equivalence relation on $\Sigma_{n}$ is the equality relation. The deck of $\pi$ is the multiset $\left\{\pi^{I}: I \in\left(\begin{array}{c}{[n]} \\ 2\end{array}\right)\right\}$ of the identification minors of $\pi$, and it is denoted by $\operatorname{deck} \pi$. If $\tau \in \Sigma_{n}$ and $\operatorname{deck} \pi=\operatorname{deck} \tau$, then $\tau$ is a reconstruction of $\pi$. If every reconstruction of $\pi$ is equal to $\pi$, then $\pi$ is reconstructible.

If $2 \leqslant n \leqslant 4$, then not all permutations of $[n]$ are reconstructible. For $n=2$, this is obvious, because there are two permutations of $\{1,2\}$ but only one permutation of $\{1\}$; hence both permutations of $\{1,2\}$ necessarily have the same deck. For $n=3$, a simple counting argument shows that there must exist permutations with the same deck: there are $3 !=6$ permutations of $\{1,2,3\}$, each has $\left(\begin{array}{l}3 \\ 2\end{array}\right)=3$ cards that are permutations of $\{1,2\}$. The number of permutations of $\{1,2\}$ is $2 !=2$. The number of 3 -element multisets over a 2-element set is 4 . Therefore, the number of possible decks is less than the number of permutations of $\{1,2,3\}$, so the non-reconstructibility of some permutations is unavoidable. Unfortunately, the same counting argument will not work when $n \geqslant 4$, so we need to take a different approach. The case $n=4$ is still quite easy to work out by hand. Indeed, in Table 1, we have enumerated all permutations of $[n]$ and their identification minors, for $2 \leqslant n \leqslant 4$. Permutations with identical decks are marked with $*$ and $\dagger$. It can be read off from the table that the permutations 1342 and 1423 have the same deck. The table also reveals the fact that, in fact, no permutation of $\{1,2,3\}$ is reconstructible.

These observations raise the question whether permutations of sets with at least five elements are reconstructible or not. We will address this question in the remainder of this paper.

\section{Reconstructibility of permutations}

In this section, we are going to establish the main result of this paper: every permutation of a finite set with at least five elements is reconstructible from its identification minors (Theorem 3.7). First, we need to introduce some notation and establish some auxiliary results. It is an easy exercise to verify the following identities involving binomial 


\begin{tabular}{|c|c|c|c|c|c|c|c|c|c|c|c|c|c|c|}
\hline \multicolumn{2}{|c|}{$n=2$} & & \multicolumn{4}{|c|}{$n=3$} & \multicolumn{8}{|c|}{$n=4$} \\
\hline$\pi$ & $\pi_{12}$ & & $\pi$ & $\pi_{12}$ & $\pi_{13}$ & $\pi_{23}$ & & $\pi$ & $\pi_{12}$ & $\pi_{13}$ & $\pi_{14}$ & $\pi_{23}$ & $\pi_{24}$ & $\pi_{34}$ \\
\hline * 12 & 1 & y & 123 & 12 & 12 & 12 & & 1234 & 123 & 123 & 123 & 123 & 123 & 123 \\
\hline * 21 & 1 & & 132 & 12 & 12 & 12 & & 1243 & 132 & 123 & 123 & 123 & 123 & 123 \\
\hline & & & 213 & 12 & 21 & 21 & & 1324 & 123 & 123 & 132 & 123 & 132 & 132 \\
\hline & & & 231 & 12 & 21 & 21 & $*$ & 1342 & 123 & 132 & 132 & 123 & 132 & 132 \\
\hline & & - & 312 & 21 & 12 & 21 & $*$ & 1423 & 132 & 132 & 123 & 132 & 123 & 132 \\
\hline & & & 321 & 21 & 12 & 21 & & 1432 & 132 & 132 & 132 & 132 & 123 & 132 \\
\hline & & & & & & & & 2134 & 123 & 213 & 213 & 213 & 213 & 213 \\
\hline & & & & & & & & 2143 & 132 & 213 & 213 & 213 & 213 & 213 \\
\hline & & & & & & & & 2314 & 123 & 213 & 231 & 213 & 231 & 231 \\
\hline & & & & & & & & 2341 & 123 & 213 & 231 & 231 & 231 & 231 \\
\hline & & & & & & & & 2413 & 132 & 231 & 213 & 231 & 213 & 231 \\
\hline & & & & & & & & 2431 & 132 & 231 & 213 & 231 & 231 & 231 \\
\hline & & & & & & & & 3124 & 213 & 123 & 312 & 213 & 312 & 312 \\
\hline & & & & & & & & 3142 & 213 & 132 & 312 & 213 & 312 & 312 \\
\hline & & & & & & & & 3214 & 213 & 123 & 321 & 213 & 321 & 321 \\
\hline & & & & & & & & 3241 & 213 & 123 & 321 & 231 & 321 & 321 \\
\hline & & & & & & & & 3412 & 231 & 132 & 312 & 231 & 321 & 312 \\
\hline & & & & & & & & 3421 & 231 & 132 & 312 & 231 & 321 & 321 \\
\hline & & & & & & & & 4123 & 312 & 312 & 123 & 312 & 213 & 312 \\
\hline & & & & & & & & 4132 & 312 & 312 & 132 & 312 & 213 & 312 \\
\hline & & & & & & & & 4213 & 312 & 321 & 123 & 321 & 213 & 321 \\
\hline & & & & & & & & 4231 & 312 & 321 & 123 & 321 & 231 & 321 \\
\hline & & & & & & & & 4312 & 321 & 312 & 132 & 321 & 231 & 312 \\
\hline & & & & & & & & 4321 & 321 & 312 & 132 & 321 & 231 & 321 \\
\hline
\end{tabular}

Table 1: The identification minors of all permutations $\pi \in \Sigma_{n}$ with $2 \leqslant n \leqslant 4$. Identical decks are indicated with $*$ and $\dagger$.

coefficients, which will be used frequently in the sequel:

$$
\begin{gathered}
\left(\begin{array}{l}
a \\
2
\end{array}\right)+a=\left(\begin{array}{c}
a+1 \\
2
\end{array}\right), \\
\left(\begin{array}{l}
a \\
2
\end{array}\right)-\left(\begin{array}{l}
b \\
2
\end{array}\right)=\left(\begin{array}{c}
a-b \\
2
\end{array}\right)+b(a-b) .
\end{gathered}
$$

Let $\pi \in \Sigma_{n}, i \in[n-1]$, and $\ell \in[n-1]$. Denote by $N(\pi, \ell, i)$ the total number of times the element $\ell$ occurs at the $i$-th position in the cards of $\pi$, i.e.,

$$
N(\pi, \ell, i):=\left|\left\{I \in\left(\begin{array}{c}
{[n]} \\
2
\end{array}\right): \pi^{I}(i)=\ell\right\}\right| .
$$

It is clear that $\sum_{i \in[n-1]} N(\pi, \ell, i)=\left(\begin{array}{l}n \\ 2\end{array}\right)=\sum_{\ell \in[n-1]} N(\pi, \ell, i)$. 
For $k \in[n]$, let

$$
\begin{aligned}
L_{\pi}(k) & :=\left\{i \in[n]: i<_{\pi} k\right\}, \\
H_{\pi}(k) & :=\left\{i \in[n]: i>k \text { and } i<_{\pi} k\right\}, \\
h_{\pi}(k) & :=\left|H_{\pi}(k)\right| .
\end{aligned}
$$

It is clear that $\left|L_{\pi}(k)\right|=\pi^{-1}(k)-1$.

For $\pi \in \Sigma_{n}$ and $k \in[n-1]$, let

$$
\begin{aligned}
& S_{1}^{\pi, k}:=\left\{I \in\left(\begin{array}{c}
{[n]} \\
2
\end{array}\right): I \subseteq[k] \text { and } I \subseteq L_{\pi}(k+1)\right\}, \\
& S_{2}^{\pi, k}:=\left\{I \in\left(\begin{array}{c}
{[n]} \\
2
\end{array}\right): I \subseteq[k] \text { and } I \nsubseteq L_{\pi}(k+1)\right\}, \\
& S_{3}^{\pi, k}:=\left\{I \in\left(\begin{array}{c}
{[n]} \\
2
\end{array}\right): I \nsubseteq[k] \text { and } k \in I \text { and } I \cap L_{\pi}(k) \neq \varnothing\right\}, \\
& S_{4}^{\pi, k}:=\left\{I \in\left(\begin{array}{c}
{[n]} \\
2
\end{array}\right): I \nsubseteq[k] \text { and } k \in I \text { and } I \cap L_{\pi}(k)=\varnothing\right\}, \\
& S_{5}^{\pi, k}:=\left\{I \in\left(\begin{array}{c}
{[n]} \\
2
\end{array}\right): I \nsubseteq[k] \text { and } k \notin I \text { and } I \subseteq L_{\pi}(k)\right\}, \\
& S_{6}^{\pi, k}:=\left\{I \in\left(\begin{array}{c}
{[n]} \\
2
\end{array}\right): I \nsubseteq[k] \text { and } k \notin I \text { and } I \nsubseteq L_{\pi}(k)\right\} .
\end{aligned}
$$

It is clear from the definition that the nonempty sets among $S_{1}^{\pi, k}, \ldots, S_{6}^{\pi, k}$ constitute a partition of $\left(\begin{array}{c}{[n]} \\ 2\end{array}\right)$. The motivation for defining this partition is that the location of $k$ in $\pi^{I}$ depends on the block $S_{i}^{\pi, k}$ to which $I$ belongs, as described by the following lemma.

Lemma 3.1. Let $\pi \in \Sigma_{n}$ and $k \in[n-1]$, and write $\alpha:=\pi^{-1}(k)$ and $\beta:=\pi^{-1}(k+1)$.

(i) If $I \in S_{1}^{\pi, k}$, then $\left(\pi^{I}\right)^{-1}(k)=\beta-1$.

(ii) If $I \in S_{2}^{\pi, k}$, then $\left(\pi^{I}\right)^{-1}(k)=\beta$.

(iii) If $I \in S_{3}^{\pi, k}$ with $I=\{k, \ell\}$, then $\left(\pi^{I}\right)^{-1}(k)=\pi^{-1}(\ell)$. (Note that $\ell<_{\pi} k$.)

(iv) If $I \in S_{4}^{\pi, k}$, then $\left(\pi^{I}\right)^{-1}(k)=\alpha$.

(v) If $I \in S_{5}^{\pi, k}$, then $\left(\pi^{I}\right)^{-1}(k)=\alpha-1$.

(vi) If $I \in S_{6}^{\pi, k}$, then $\left(\pi^{I}\right)^{-1}(k)=\alpha$.

Proof. Assume first that $I \in S_{1}^{\pi, k} \cup S_{2}^{\pi, k}$. Then $I \subseteq[k]$, so the element $k+1$ at position $\beta$ is decreased by 1 (so that it becomes $k$ ) upon formation of the minor $\pi^{I}$. If $I \in S_{1}^{\pi, k}$, then $I \subseteq L_{\pi}(k+1)$, so the element that gets deleted upon formation of the minor $\pi^{I}$ is located to the left from position $\beta$, and we conclude that $\left(\pi^{I}\right)^{-1}(k)=\beta-1$ whenever $I \in S_{1}^{\pi, k}$. Otherwise (i.e., if $\left.I \in S_{2}^{\pi, k}\right)$ the element that gets deleted is to the right from position $\beta$, and we conclude that $\left(\pi^{I}\right)^{-1}(k)=\beta$ whenever $I \in S_{2}^{\pi, k}$.

Assume then that $I \in S_{3}^{\pi, k} \cup S_{4}^{\pi, k}$. Then $I \nsubseteq[k]$ and $k \in I$, that is, $I=\{k, \ell\}$ for some $\ell \in[n] \backslash[k]$. Consequently, the element $k$ at position $\alpha$ is not decreased nor does it get deleted upon formation of the minor $\pi^{I}$, but it may be swapped with $\ell$ before $\ell$ gets deleted. If $\ell<_{\pi} k$ (i.e., if $I \in S_{3}^{\pi, k}$ ), then $k$ and $\ell$ are first swapped before deleting 
$\ell$, and we conclude that $\left(\pi^{I}\right)^{-1}(k)=\pi^{-1}(\ell)$ whenever $I=\{k, \ell\} \in S_{3}^{\pi, k}$. If $k<_{\pi} \ell$ (i.e., if $\left.I \in S_{4}^{\pi, k}\right)$, then $k$ remains at its place at position $\alpha$ and $\ell$ gets deleted, and we conclude that $\left(\pi^{I}\right)^{-1}(k)=\alpha$ whenever $I \in S_{4}^{\pi, k}$.

Assume then that $I \in S_{5}^{\pi, k} \cup S_{6}^{\pi, k}$. Then $I \nsubseteq[k]$ and $k \notin I$. This implies that $I$ contains an element strictly greater than $k$. Therefore the element $k$ at position $\alpha$ is not decreased by 1 upon formation of the minor $\pi^{I}$. If $I \in S_{5}^{\pi, k}$, then the element that gets deleted upon formation of the minor $\pi^{I}$ is located to the left from position $\alpha$, and we conclude that $\left(\pi^{I}\right)^{-1}(k)=\alpha-1$. Otherwise (i.e., if $I \in S_{6}^{\pi, k}$ ) the element that gets deleted is to the right from position $\alpha$, and we conclude that $\left(\pi^{I}\right)^{-1}(k)=\alpha$ whenever $I \in S_{6}^{\pi, k}$.

Lemma 3.2. Let $\pi \in \Sigma_{n}$ and $k \in[n-1]$, and write $\alpha:=\pi^{-1}(k)$ and $\beta:=\pi^{-1}(k+1)$. Then the cardinalities of the sets $S_{i}^{\pi, k}(1 \leqslant i \leqslant 6)$ are the following:

$$
\begin{aligned}
\left|S_{1}^{\pi, k}\right| & =\left(\begin{array}{c}
\beta-1-h_{\pi}(k+1) \\
2
\end{array}\right), \\
\left|S_{2}^{\pi, k}\right| & =\left(\begin{array}{c}
k \\
2
\end{array}\right)-\left|S_{1}^{\pi, k}\right|, \\
\left|S_{3}^{\pi, k}\right| & =h_{\pi}(k), \\
\left|S_{4}^{\pi, k}\right| & =n-k-\left|S_{3}^{\pi, k}\right|, \\
\left|S_{5}^{\pi, k}\right| & =\left(\begin{array}{c}
\alpha-1 \\
2
\end{array}\right)-\left(\begin{array}{c}
\alpha-1-h_{\pi}(k) \\
2
\end{array}\right), \\
\left|S_{6}^{\pi, k}\right| & =\left(\begin{array}{c}
n-k \\
2
\end{array}\right)+(k-1)(n-k)-\left|S_{5}^{\pi, k}\right| .
\end{aligned}
$$

Furthermore, $\left|S_{1}^{\pi, k}\right|+\left|S_{2}^{\pi, k}\right|=\left(\begin{array}{c}k \\ 2\end{array}\right),\left|S_{3}^{\pi, k}\right|+\left|S_{4}^{\pi, k}\right|=n-k$, and $\left|S_{5}^{\pi, k}\right|+\left|S_{6}^{\pi, k}\right|=\left(\begin{array}{c}n-k \\ 2\end{array}\right)+$ $(k-1)(n-k)$.

Proof. The set $S_{1}^{\pi, k}$ is the set of all 2-element subsets of $[k] \cap L_{\pi}(k+1)$. It is easy to see that $[k] \cap L_{\pi}(k+1)=L_{\pi}(k+1) \backslash H_{\pi}(k+1)$. Since $H_{\pi}(k+1) \subseteq L_{\pi}(k+1)$, we have $\left|L_{\pi}(k+1) \backslash H_{\pi}(k+1)\right|=\beta-1-h_{\pi}(k+1)$. The claim about $\left|S_{1}^{\pi, k}\right|$ thus follows.

Observe that $S_{1}^{\pi, k} \cup S_{2}^{\pi, k}=\left\{I \in\left(\begin{array}{c}{[n]} \\ 2\end{array}\right): I \subseteq[k]\right\}=\left(\begin{array}{c}{[k]} \\ 2\end{array}\right)$. Since $S_{1}^{\pi, k}$ and $S_{2}^{\pi, k}$ are disjoint, we have $\left|S_{1}^{\pi, k}\right|+\left|S_{2}^{\pi, k}\right|=\left(\begin{array}{c}k \\ 2\end{array}\right)$, and the claim about $\left|S_{2}^{\pi, k}\right|$ follows.

The condition $I \nsubseteq[k]$ and $k \in I$ is equivalent to the condition that $I=\{k, \ell\}$ for some $\ell \in[n] \backslash[k]$. Therefore $\left|S_{3}^{\pi, k}\right|+\left|S_{4}^{\pi, k}\right|=n-k$. The set $S_{3}^{\pi, k}$ contains exactly those couples $I=\{k, \ell\}$ where $\ell>k$ and $\ell<_{\pi} k$, that is, $\ell \in H_{\pi}(k)$. Thus $\left|S_{3}^{\pi, k}\right|=h_{\pi}(k)$, and the claim about $\left|S_{4}^{\pi, k}\right|$ follows.

The set $S_{5}^{\pi, k}$ is the set of all 2-element subsets of $L_{\pi}(k)$ that are not subsets of $[k]$. We have $[k] \cap L_{\pi}(k)=L_{\pi}(k) \backslash H_{\pi}(k)$, and since $H_{\pi}(k) \subseteq L_{\pi}(k)$, we have $\left|L_{\pi}(k) \backslash H_{\pi}(k)\right|=$ $\alpha-1-h_{\pi}(k)$. Consequently,

$$
\left|S_{5}^{\pi, k}\right|=\left(\begin{array}{c}
\alpha-1 \\
2
\end{array}\right)-\left(\begin{array}{c}
\alpha-1-h_{\pi}(k) \\
2
\end{array}\right)
$$


Finally, observe that $S_{5}^{\pi, k} \cup S_{6}^{\pi, k}=\left\{I \in\left(\begin{array}{c}{[n]} \\ 2\end{array}\right): I \nsubseteq[k]\right.$ and $\left.k \notin I\right\}$. The cardinality of this set is $\left(\begin{array}{c}n-k \\ 2\end{array}\right)+(k-1)(n-k)$. Since $S_{5}^{\pi, k}$ and $S_{6}^{\pi, k}$ are disjoint, we have $\left|S_{5}^{\pi, k}\right|+\left|S_{6}^{\pi, k}\right|=$ $\left(\begin{array}{c}n-k \\ 2\end{array}\right)+(k-1)(n-k)$, and the claim about $\left|S_{6}^{\pi, k}\right|$ follows.

Lemma 3.3. Let $\pi \in \Sigma_{n}$ and $k \in[n-1]$. Write $\alpha:=\pi^{-1}(k)$. Then both $S_{4}^{\pi, k}$ and $S_{6}^{\pi, k}$ are empty if and only if $n=\alpha$.

Proof. Assume that $S_{4}^{\pi, k}=S_{6}^{\pi, k}=\varnothing$. Lemma 3.2 then implies that $n-k=h_{\pi}(k)$ and $\left(\begin{array}{c}n-k \\ 2\end{array}\right)+(k-1)(n-k)=\left(\begin{array}{c}\alpha-1 \\ 2\end{array}\right)-\left(\begin{array}{c}\alpha-1-h_{\pi}(k) \\ 2\end{array}\right)$. Substituting $n-k$ for $h_{\pi}(k)$ in the latter identity and then applying identity (2), we obtain

$$
\begin{aligned}
\left(\begin{array}{c}
n-k \\
2
\end{array}\right)+(k-1)(n-k) & =\left(\begin{array}{c}
\alpha-1 \\
2
\end{array}\right)-\left(\begin{array}{c}
\alpha-1-n+k \\
2
\end{array}\right) \\
& =\left(\begin{array}{c}
n-k \\
2
\end{array}\right)+(\alpha-1-n+k)(n-k),
\end{aligned}
$$

which implies $(\alpha-n)(n-k)=0$, that is, $n=\alpha$ or $n=k$. The latter is not possible, because we are assuming that $k \in[n-1]$, so we conclude that $n=\alpha$.

If $n=\alpha$, then $\pi(n)=k$, and we have $i<_{\pi} k$ for all $i \in[n-1] \backslash\{k\}$, i.e., $L_{\pi}(k)=$ $[n-1] \backslash\{k\}$. Therefore $I \cap L_{\pi}(k) \neq \varnothing$ for all $I \in\left(\begin{array}{c}{[n]} \\ 2\end{array}\right)$, and $I \subseteq L_{\pi}(k)$ for all $I \in\left(\begin{array}{c}{[n]} \\ 2\end{array}\right)$ with $k \notin I$. This means in particular that no set $I \in\left(\begin{array}{c}{[n]} \\ 2\end{array}\right)$ satisfies the defining conditions of $S_{4}^{\pi, k}$ and $S_{6}^{\pi, k}$, i.e., $S_{4}^{\pi, k}=S_{6}^{\pi, k}=\varnothing$.

For $n \in \mathbb{N}_{+}$and $\alpha, \beta \in[n]$ with $\alpha \neq \beta$, define condition $C(\alpha, \beta)$ as indicated in Table 2. The couples $(\alpha, \beta)$ and the corresponding conditions $C(\alpha, \beta)$ are partitioned into eleven types: $(\mathrm{A}), \ldots,(\mathrm{K})$. The symbol $T$ occurring in the conditions will be given an interpretation in Lemma 3.4.

Lemma 3.4. Let $n \geqslant 5$ and $\pi \in \Sigma_{n}$. Let $T:=\{i \in[n-1]: N(\pi, n-1, i)>0\}$.

(i) For any $\alpha, \beta \in[n]$ with $\alpha \neq \beta$, unless $n=7$ and $(\alpha, \beta) \in\{(4,6),(7,5)\}$, we have $\pi^{-1}(n-1)=\alpha$ and $\pi^{-1}(n)=\beta$ if and only if condition $C(\alpha, \beta)$ holds.

(ii) For $n=7$, we have $\pi^{-1}(n-1)=4$ and $\pi^{-1}(n)=6$ if and only if the following three statements are true:

(a) condition $C(4,6)$ (of type $(\mathrm{B}))$ holds,

(b) $\left|\left\{I \in\left(\begin{array}{c}{[n]} \\ 2\end{array}\right): 5<_{\pi^{I}} 6\right\}\right| \geqslant 11$, and

(c) if the inequality in (b) holds as an equality, then:

(c.1) $N(\pi, c, 6)=0$ for every $c \in\{1,2,3\}$, and

(c.2) there exists $d \in[n]$ such that $\pi^{I}(5)=d$ for every $I \in\left(\begin{array}{c}{[n]} \\ 2\end{array}\right)$ satisfying $\pi^{I}(4)=6$ and $\pi^{I}(6)=5$.

(iii) For $n=7$, we have $\pi^{-1}(n-1)=7$ and $\pi^{-1}(n)=5$ if and only if the following three statements are true: 


\begin{tabular}{|c|c|c|}
\hline type & $(\alpha, \beta)$ & $C(\alpha, \beta)$ \\
\hline (A) & $\begin{array}{l}1 \leqslant \alpha \leqslant n-2 \\
\alpha=\beta-1\end{array}$ & $\begin{array}{l}T=\{p, q\} \text { with } p=\alpha=\beta-1, q=\beta \\
N(\pi, n-1, p)=\left(\begin{array}{c}p \\
2\end{array}\right)+n-1 \\
N(\pi, n-1, q)=\left(\begin{array}{c}n-1 \\
2\end{array}\right)-\left(\begin{array}{c}q-1 \\
2\end{array}\right)\end{array}$ \\
\hline (B) & $\begin{array}{l}1 \leqslant \alpha \leqslant n-3 \\
\alpha<\beta-1 \\
\beta \leqslant n-1\end{array}$ & $\begin{array}{l}T=\{p, q, r\} \text { with } p=\alpha, q=\beta-1, r=\beta \\
N(\pi, n-1, p)=n-1 \\
N(\pi, n-1, q)=\left(\begin{array}{c}q \\
2\end{array}\right) \\
N(\pi, n-1, r)=\left(\begin{array}{c}n-1 \\
2\end{array}\right)-\left(\begin{array}{c}r-1 \\
2\end{array}\right)\end{array}$ \\
\hline (C) & $\begin{array}{l}1 \leqslant \alpha \leqslant n-2 \\
\beta=n\end{array}$ & $\begin{array}{l}T=\{p, q\} \text { with } p=\alpha, q=\beta-1=n-1 \\
N(\pi, n-1, p)=n-1 \\
N(\pi, n-1, q)=\left(\begin{array}{c}q \\
2\end{array}\right)=\left(\begin{array}{c}n-1 \\
2\end{array}\right)\end{array}$ \\
\hline (D) & $\begin{array}{l}\alpha=n-1 \\
\beta=n\end{array}$ & $T=\{p\}$ with $p=n-1$ \\
\hline$(\mathrm{E})$ & $\begin{array}{l}1 \leqslant \beta \leqslant 2 \\
\beta=\alpha-1\end{array}$ & $\begin{array}{l}T=\{p, q\} \text { with } p=\beta=\alpha-1, q=\alpha \\
N(\pi, n-1, p)=\left(\begin{array}{c}n-1 \\
2\end{array}\right)+p \\
N(\pi, n-1, q)=n-q\end{array}$ \\
\hline$(\mathrm{F})$ & $\begin{array}{l}1 \leqslant \beta \leqslant 2 \\
\beta<\alpha-1 \\
\alpha \leqslant n-1\end{array}$ & $\begin{array}{l}T=\{p, q, r\} \text { with } p=\beta, q=\alpha-1, r=\alpha \\
N(\pi, n-1, p)=\left(\begin{array}{c}n-1 \\
2\end{array}\right)+1 \\
N(\pi, n-1, q)=q-1 \\
N(\pi, n-1, r)=n-r\end{array}$ \\
\hline$(\mathrm{G})$ & $\begin{array}{l}1 \leqslant \beta \leqslant 2 \\
\alpha=n\end{array}$ & $\begin{array}{l}T=\{p, q\} \text { with } p=\beta, q=n-1 \\
N(\pi, n-1, p)=\left(\begin{array}{c}n-1 \\
2\end{array}\right)+1 \\
N(\pi, n-1, q)=n-2\end{array}$ \\
\hline$(\mathrm{H})$ & $\begin{array}{l}3 \leqslant \beta \leqslant n-2 \\
\beta=\alpha-1\end{array}$ & $\begin{array}{l}T=\{p, q, r\} \text { with } p=\beta-1, q=\beta=\alpha-1, r=\alpha \\
N(\pi, n-1, p)=\left(\begin{array}{c}p \\
2\end{array}\right) \\
N(\pi, n-1, q)=\left(\begin{array}{c}n-1 \\
2\end{array}\right)-\left(\begin{array}{c}q-1 \\
2\end{array}\right)+q \\
N(\pi, n-1, r)=n-r\end{array}$ \\
\hline (I) & $\begin{array}{l}3 \leqslant \beta \leqslant n-3 \\
\beta<\alpha-1 \\
\alpha \leqslant n-1\end{array}$ & $T=\{p, q, r, s\}$ with $p=\beta-1, q=\beta, r=\alpha-1, s=\alpha$ \\
\hline$(\mathrm{J})$ & $\begin{array}{l}3 \leqslant \beta \leqslant n-2 \\
\alpha=n\end{array}$ & $\begin{array}{l}T=\{p, q, r\} \text { with } p=\beta-1, q=\beta, r=\alpha-1=n-1 \\
N(\pi, n-1, p)=\left(\begin{array}{c}p \\
2\end{array}\right) \\
N(\pi, n-1, q)=\left(\begin{array}{c}n-1 \\
2\end{array}\right)-\left(\begin{array}{c}q-1 \\
2\end{array}\right)+1 \\
N(\pi, n-1, r)=n-2\end{array}$ \\
\hline$(\mathrm{K})$ & $\begin{array}{l}\beta=n-1 \\
\alpha=n\end{array}$ & $\begin{array}{l}T=\{p, q\} \text { with } p=n-2, q=n-1 \\
N(\pi, n-1, p)=\left(\begin{array}{c}n-2 \\
2\end{array}\right) \\
N(\pi, n-1, q)=\left(\begin{array}{c}n \\
2\end{array}\right)-\left(\begin{array}{c}n-2 \\
2\end{array}\right)\end{array}$ \\
\hline
\end{tabular}

Table 2: Conditions $C(\alpha, \beta)$ of Lemma 3.4. 
(a) condition $C(7,5)$ (of type $(\mathrm{J})$ ) holds,

(b) $\left|\left\{I \in\left(\begin{array}{c}{[n]} \\ 2\end{array}\right): 5<_{\pi^{I}} 6\right\}\right| \leqslant 11$, and

(c) if the inequality in (b) holds as an equality, then:

(c.1) there exists $c \in\{1,2,3\}$ such that $N(\pi, c, 6)>0$, or

(c.2) there does not exist any $d \in[n]$ such that $\pi^{I}(5)=d$ for every $I \in\left(\begin{array}{c}{[n]} \\ 2\end{array}\right)$ satisfying $\pi^{I}(4)=6$ and $\pi^{I}(6)=5$.

Proof. We are first going to prove the forward implications of each of (i), (ii), and (iii). Write $\alpha:=\pi^{-1}(n-1)$ and $\beta:=\pi^{-1}(n)$. Let us apply Lemma 3.2 to determine the cardinalities of the sets $S_{1}^{\pi, k}, \ldots, S_{6}^{\pi, k}$ for $k=n-1$. We clearly have

$$
h_{\pi}(n)=0 \quad \text { and } \quad h_{\pi}(n-1)= \begin{cases}0, & \text { if } n-1<_{\pi} n \\ 1, & \text { if } n<_{\pi} n-1 .\end{cases}
$$

Hence the formulas of Lemma 3.2 yield the following

$$
\begin{aligned}
\left|S_{1}^{\pi, k}\right| & =\left(\begin{array}{c}
\beta-1 \\
2
\end{array}\right), & \left|S_{2}^{\pi, k}\right|=\left(\begin{array}{c}
n-1 \\
2
\end{array}\right)-\left(\begin{array}{c}
\beta-1 \\
2
\end{array}\right), \\
\left|S_{3}^{\pi, k}\right| & = \begin{cases}0, & \text { if } n-1<_{\pi} n, \\
1, & \text { if } n<_{\pi} n-1,\end{cases} & \left|S_{4}^{\pi, k}\right|= \begin{cases}1, & \text { if } n-1<_{\pi} n, \\
0, & \text { if } n<_{\pi} n-1,\end{cases} \\
\left|S_{5}^{\pi, k}\right| & = \begin{cases}0, & \text { if } n-1<_{\pi} n, \\
\alpha-2, & \text { if } n<_{\pi} n-1,\end{cases} & \left|S_{6}^{\pi, k}\right|= \begin{cases}n-2, & \text { if } n-1<_{\pi} n, \\
n-\alpha, & \text { if } n<_{\pi} n-1 .\end{cases}
\end{aligned}
$$

(In order to see this, note that identity (1) gives $\left(\begin{array}{c}\alpha-1 \\ 2\end{array}\right)-\left(\begin{array}{c}\alpha-2 \\ 2\end{array}\right)=\alpha-2$ and $\left(\begin{array}{c}n \\ 2\end{array}\right)-\left(\begin{array}{c}n-1 \\ 2\end{array}\right)-$ $(n-(n-1))=n-2$.

In the case when $n<_{\pi} n-1$, the set $S_{3}^{\pi, k}$ is nonempty, namely $S_{3}^{\pi, k}=\{\{n-1, n\}\}$, and we have $\pi^{I}(\beta)=n-1$ for $I=\{n-1, n\}$. Thus, using Lemma 3.1, we get the numbers $N(\pi, n-1, i)$ for $i \in\{\alpha-1, \alpha, \beta-1, \beta\}$ as indicated in Table 3. For $i \in$ $[n] \backslash\{\alpha-1, \alpha, \beta-1, \beta\}$, we have $N(\pi, n-1, i)=0$. In the case when $\alpha=\beta-1$ or $\beta=\alpha-1$, we must take the sum of the respective two numbers: if $\alpha=\beta-1$ then $N(\pi, n-1, \alpha)=\left(\begin{array}{c}\beta-1 \\ 2\end{array}\right)+n-1=\left(\begin{array}{c}\alpha \\ 2\end{array}\right)+n-1$; if $\beta=\alpha-1$ then $N(\pi, n-1, \beta)=$ $\left(\begin{array}{c}n-1 \\ 2\end{array}\right)-\left(\begin{array}{c}\beta-1 \\ 2\end{array}\right)+\alpha-1=\left(\begin{array}{c}n-1 \\ 2\end{array}\right)-\left(\begin{array}{c}\beta-1 \\ 2\end{array}\right)+\beta$. Straightforward calculations with the data from Table 3 then give the numbers $N(\pi, n-1, i)$ shown in Table 2 . This proves the forward implication of (i), as well as the necessity of condition (a) in (ii) and (iii).

We still need to establish the necessity of conditions (b) and (c) in statements (ii) and (iii). We will do this in parallel for both (ii) and (iii). Let $n=7$ and let $\sigma, \tau \in \Sigma_{7}$ be permutations satisfying $\sigma(4)=6, \sigma(6)=7, \tau(7)=6, \tau(5)=7$. For every $I \in\left(\begin{array}{c}{[n]} \\ 2\end{array}\right)$ such that $I \subseteq[n] \backslash\{6,7\}$, we have $5<_{\sigma^{I}} 6$ and $6<_{\tau^{I}} 5$. Moreover, if $I=\{\sigma(4), \sigma(7)\}=$ $\{6, \sigma(7)\}$, then $\sigma^{I}(6)=6$, so we have $5<_{\sigma^{I}} 6$ also in this case. Therefore, out of the 21 cards of $\sigma$, at least $\left(\begin{array}{l}5 \\ 2\end{array}\right)+1=11$ satisfy $5<_{\sigma^{I}} 6$. Similarly, out of the 21 cards of $\tau$, at least $\left(\begin{array}{l}5 \\ 2\end{array}\right)=10$ satisfy $6<_{\tau^{I}} 5$ and hence at most 11 satisfy $5<_{\sigma^{I}} 6$. This proves the necessity of (b). 


\begin{tabular}{|c|c|cccc|}
\hline$\alpha<\beta$ & $i$ & $\alpha-1$ & $\alpha$ & $\beta-1$ & $\beta$ \\
$n-1<_{\pi} n$ & $N(\pi, n-1, i)$ & 0 & $n-1$ & $\left(\begin{array}{c}\beta-1 \\
2\end{array}\right)$ & $\left(\begin{array}{c}n-1 \\
2\end{array}\right)-\left(\begin{array}{c}\beta-1 \\
2\end{array}\right)$ \\
\hline$\beta<\alpha$ & $i$ & $\beta-1$ & $\beta$ & $\alpha-1$ & $\alpha$ \\
\cline { 2 - 6 }$n<_{\pi} n-1$ & $N(\pi, n-1, i)$ & $\left(\begin{array}{c}\beta-1 \\
2\end{array}\right)$ & $\left(\begin{array}{c}n-1 \\
2\end{array}\right)-\left(\begin{array}{c}\beta-1 \\
2\end{array}\right)+1$ & $\alpha-2$ & $n-\alpha$ \\
\hline
\end{tabular}

Table 3: Number of occurrences of $n-1$ in different positions in the cards of $\pi$.

Assume from now on that the inequalities of statement (b) in both (ii) and (iii) hold as equalities, that is, exactly 11 cards $\sigma^{I}$ satisfy $5<_{\sigma^{I}} 6$ and exactly 11 cards $\tau^{I}$ satisfy $5<_{\tau^{I}} 6$. Consequently, $5<_{\sigma^{I}} 6$ if and only if $I \subseteq[n] \backslash\{6,7\}$ or $I=\{6, \sigma(7)\}$. Therefore, if $I=\{\sigma(1), 6\}$, then we have $6<_{\sigma^{I}} 5$ and $\sigma^{I}(5)=6$. This is possible only if $\sigma^{I}(6)=5$, which in turn implies that $\sigma(7)=5$.

Let us write down the minors of $\sigma$ and $\tau$, providing as much details as we can deduce from the known facts: $\sigma(4)=6, \sigma(6)=7, \sigma(7)=5, \tau(5)=7, \tau(7)=6$. See the first two columns of Table 4 . The rows are labeled as $i j$, where $i, j \in[7]$ with $i<j$. The row then represents the minor $\sigma^{I}$ with $I=\{\sigma(i), \sigma(j)\}$ or $\tau^{I}$ with $I=\{\tau(i), \tau(j)\}$. In the bottom part of the tables we have counted the number of times each element of [6] occurs at each position in the table. The blanks in the table represent unknown values. Note that $N(\sigma, c, 6)=0$ for every $c \in\{1,2,3\}$.

For every $I \in\left(\begin{array}{c}{[n]} \\ 2\end{array}\right)$ with $7 \in I$, we have that $\tau^{I}(6)=\tau(6)$. We cannot have $\tau(6)=5$, because then all cards $\tau^{I}$ have the property that $6<_{\tau^{I}} 5$, contradicting the assumption that the equality case of the inequality in (b) holds. If $\tau(6)=c$ for some $c \in\{1,2,3\}$, then $N(\tau, c, 6)>0$.

Therefore, it only remains to consider the case $\tau(6)=4$. Having fixed this value, we can complement the information about the cards of $\tau$; see the rightmost column of Table 4. Each one of the entries marked with $*$ is either 3 or 4 ; three of them are 3's and three of them are 4's. Exactly which ones are 3 and which ones are 4 depends on which one of the three numbers $1,2,3$ is mapped to 5 by $\tau$.

Observe that the six minors $\sigma^{I}$ of $\sigma$ that satisfy $\sigma^{I}(4)=6$ and $\sigma^{I}(6)=5$ are precisely the ones with $\sigma(6)=7 \in I$. It follows that for each such $I, \sigma^{I}(5)=\sigma(5)$. On the other hand, among the six minors $\tau^{I}$ of $\tau$ that satisfy $\tau^{I}(4)=6$ and $\tau^{I}(6)=5$, exactly three satisfy $\tau^{I}(5)=3$ and exactly three satisfy $\tau^{I}(5)=4$ (recall from the previous paragraph the discussion about the entries $*$ ).

We have now established that the conditions of statements (i), (ii), and (iii) are necessary for $\pi^{-1}(n-1)=\alpha$ and $\pi^{-1}(n)=\beta$. Since all possible values of $\alpha, \beta$, and $n$ are covered by statements (i), (ii), and (iii), sufficiency of these conditions will follow if we show that the conditions are mutually exclusive. Observe first that the conditions of statements (ii) and (iii) clearly cannot both hold simultaneously. It is thus enough to show that the conditions $C(\alpha, \beta)$ with $\alpha, \beta \in[n], \alpha \neq \beta$, are mutually exclusive, with the single exception that conditions $C(4,6)$ and $C(7,5)$ are identical when $n=7$.

So, let $\alpha, \beta, \alpha^{\prime}, \beta^{\prime} \in[n]$ with $\alpha \neq \beta, \alpha^{\prime} \neq \beta^{\prime}$ and $(\alpha, \beta) \neq\left(\alpha^{\prime}, \beta^{\prime}\right)$, and assume that $C(\alpha, \beta)$ and $C\left(\alpha^{\prime}, \beta^{\prime}\right)$ hold simultaneously. Then, first of all, both conditions must specify 


\begin{tabular}{|c|c|c|c|c|c|c|c|c|c|c|c|c|c|c|c|}
\hline \multirow[b]{3}{*}{$i j$} & \multicolumn{4}{|c|}{$\left(\begin{array}{rr}1234567 \\
6 & 75\end{array}\right)$} & \multicolumn{5}{|c|}{$=\left(\begin{array}{rr}1234567 \\
7 & 6\end{array}\right)$} & \multicolumn{6}{|c|}{$\left(\begin{array}{r}1234567 \\
746\end{array}\right)$} \\
\hline & \multicolumn{4}{|c|}{$\sigma^{I}$} & \multicolumn{6}{|c|}{$\tau^{I}$} & \multicolumn{5}{|c|}{$\tau^{I}$} \\
\hline & 12 & 3 & 4 & 56 & $i j$ & 12 & 3 & 45 & 6 & $i j$ & $\begin{array}{ll}12 \\
\end{array}$ & 3 & 4 & 5 & 6 \\
\hline 12 & & 5 & & 64 & 12 & & & 6 & 5 & 12 & & & 6 & $*$ & 5 \\
\hline 13 & & 5 & & 6 & 13 & & & 6 & 5 & 13 & & & 6 & $*$ & 5 \\
\hline 14 & & & & 65 & 14 & & & 6 & 5 & 14 & & & 6 & * & 5 \\
\hline 15 & & & 5 & 64 & 15 & & & & 6 & 15 & & & & 4 & 6 \\
\hline 16 & & & 6 & 5 & 16 & & & 6 & 5 & 16 & & & & 6 & 5 \\
\hline 17 & & & 5 & 6 & 17 & & & 6 & & 17 & & & & 6 & 4 \\
\hline 23 & & 5 & & 6 & 23 & & & 6 & 5 & 23 & & & 6 & $*$ & 5 \\
\hline 24 & & & 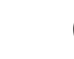 & 65 & 24 & & & 6 & 5 & 24 & & & 6 & $*$ & 5 \\
\hline 25 & & & 5 & 6 & 25 & & & & 6 & 25 & & & & 4 & 6 \\
\hline 26 & & & 6 & 5 & 26 & & & 6 & 5 & 26 & & & & 6 & 5 \\
\hline 27 & & & 5 & 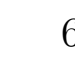 & 27 & & & 6 & & 27 & & & & 6 & 4 \\
\hline 34 & & & & 65 & 34 & & & 6 & 5 & 34 & & & 6 & * & 5 \\
\hline 35 & & & 5 & 64 & 35 & & & & 6 & 35 & & & & 4 & 6 \\
\hline 36 & & & 6 & 5 & 36 & & & 6 & 5 & 36 & & & & 6 & 5 \\
\hline 37 & & & 5 & 6 & 37 & & & 6 & & 37 & & & & 6 & 4 \\
\hline 45 & & & & 6 & 45 & & & & 6 & 45 & & & & 4 & 6 \\
\hline 46 & & & 6 & 5 & 46 & & & 6 & 5 & 46 & & & & 6 & 5 \\
\hline 47 & & & 5 & 6 & 47 & & & 6 & & 47 & & & & 6 & 4 \\
\hline 56 & & & 6 & 5 & 56 & & & & 6 & 56 & & & & 4 & 6 \\
\hline 57 & & & 5 & 6 & 57 & & & 6 & & 57 & & & & 6 & 4 \\
\hline 67 & & & 6 & 5 & 67 & & & 6 & & 67 & & & & 6 & 4 \\
\hline 1: & & & & 0 & 1: & & & & & 1: & & & & 0 & 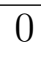 \\
\hline $2:$ & & & & 0 & $2:$ & & & & & $2:$ & & & & 0 & 0 \\
\hline 3: & & & & c & $3:$ & & & & & 3: & & & & 3 & 0 \\
\hline 4: & & & & 6 & 4: & & & & & 4: & & & & 8 & 6 \\
\hline 5: & $\begin{array}{ll}0 & 0\end{array}$ & 3 & 8 & 010 & 5: & & & & 10 & 5: & & & & 0 & 10 \\
\hline $6:$ & $\begin{array}{ll}0 & 0\end{array}$ & 0 & & 105 & $6:$ & $\begin{array}{ll}0 & 0\end{array}$ & 0 & 610 & 5 & $6:$ & $\begin{array}{ll}0 & 0\end{array}$ & 0 & & 0 & 5 \\
\hline
\end{tabular}

Table 4: Minors of permutations $\sigma$ and $\tau$. Each row $i j$ represents $\sigma^{I}$ with $I=\{\sigma(i), \sigma(j)\}$ or $\tau^{I}$ with $I=\{\tau(i), \tau(j)\}$.

the same set $T$. Therefore it is necessary that the set $T$ specified by condition $C(\alpha, \beta)$ has the same cardinality as the set $T$ specified by condition $C\left(\alpha^{\prime}, \beta^{\prime}\right)$. Thus we have four possibilities: either $(\alpha, \beta)$ and $\left(\alpha^{\prime}, \beta^{\prime}\right)$ are both of type (D) of Table 2 (in which case $|T|=1$ ), or the types of both are among (A), (C), (E), (G), (K) (in which case $|T|=2$ ), or the types of both are among $(\mathrm{B}),(\mathrm{F}),(\mathrm{H}),(\mathrm{J})$ (in which case $|T|=3$ ), or they are both of type (I) (in which case $|T|=4$ ). Furthermore, it is easy to see that if $(\alpha, \beta)$ and 
$\left(\alpha^{\prime}, \beta^{\prime}\right)$ are of the same type of Table 2 , then the set $T$ specified by condition $C(\alpha, \beta)$ is distinct from the set $T$ specified by $C\left(\alpha^{\prime}, \beta^{\prime}\right)$. We can therefore assume that $(\alpha, \beta)$ and $\left(\alpha^{\prime}, \beta^{\prime}\right)$ are of distinct types (and hence $2 \leqslant|T| \leqslant 3$ ). The remainder of this proof is a case-by-case analysis that leads to a contradiction in each case, except for the case when $n=7$ and $\left\{(\alpha, \beta),\left(\alpha^{\prime}, \beta^{\prime}\right)\right\}=\{(4,6),(7,5)\}$.

Consider first the case when $|T|=2$. Assume that $T=\{p, q\}$ with $p<q$. Suppose first that one of $(\alpha, \beta)$ and $\left(\alpha^{\prime}, \beta^{\prime}\right)$ is of type $(\mathrm{A})$ and the other is of type $(\mathrm{C})$. Without loss of generality, let us assume that $(\alpha, \beta)$ is of type $(\mathrm{A})$ and $\left(\alpha^{\prime}, \beta^{\prime}\right)$ is of type $(\mathrm{C})$. By $C\left(\alpha^{\prime}, \beta^{\prime}\right)$ we have $q=n-1$ and by $C(\alpha, \beta)$ we have $p=q-1=n-2$. Then $\left(\begin{array}{c}n-2 \\ 2\end{array}\right)+n-1=N(\pi, n-1, p)=n-1$, so $\left(\begin{array}{c}n-2 \\ 2\end{array}\right)=0$. This implies that $n \leqslant 3$, which contradicts our assumption that $n \geqslant 5$.

Suppose then that $(\alpha, \beta)$ and $\left(\alpha^{\prime}, \beta^{\prime}\right)$ are of types $(\mathrm{A})$ and $(\mathrm{E})$, respectively. Since a condition of type $(\mathrm{E})$ holds, we have $1 \leqslant p \leqslant 2$, which implies $\left(\begin{array}{l}p \\ 2\end{array}\right)=p-1$. Then $p-1+n-1=N(\pi, n-1, p)=\left(\begin{array}{c}n-1 \\ 2\end{array}\right)+p$, which implies $\left(\begin{array}{c}n-1 \\ 2\end{array}\right)=n-2$. The solution of this equation is $n=2$ or $n=3$. These solutions are not feasible, because we are assuming that $n \geqslant 5$.

Suppose that $(\alpha, \beta)$ and $\left(\alpha^{\prime}, \beta^{\prime}\right)$ are of types $(\mathrm{A})$ and $(\mathrm{G})$, respectively. By $C\left(\alpha^{\prime}, \beta^{\prime}\right)$ we have $q=n-1$ and by $C(\alpha, \beta)$ we have $p=q-1=n-2$. Since a condition of type $(\mathrm{G})$ holds, we have $1 \leqslant p \leqslant 2$, which implies that $3 \leqslant n \leqslant 4$. This contradicts our assumption that $n \geqslant 5$. In a similar way, we reach a contradiction if we suppose that $(\alpha, \beta)$ and $\left(\alpha^{\prime}, \beta^{\prime}\right)$ are of types $(\mathrm{C})$ and $(\mathrm{E})$, respectively.

Suppose that $(\alpha, \beta)$ and $\left(\alpha^{\prime}, \beta^{\prime}\right)$ are of types $(\mathrm{A})$ and $(\mathrm{K})$, respectively. By $C\left(\alpha^{\prime}, \beta^{\prime}\right)$ we have $p=n-2$ and $q=n-1$. Then $\left(\begin{array}{c}n-2 \\ 2\end{array}\right)+n-1=N(\pi, n-1, p)=\left(\begin{array}{c}n-2 \\ 2\end{array}\right)$, which implies that $n=1$. This contradicts our assumption that $n \geqslant 5$.

Suppose that $(\alpha, \beta)$ and $\left(\alpha^{\prime}, \beta^{\prime}\right)$ are of types $(\mathrm{C})$ and $(\mathrm{G})$, respectively. Then $n-1=$ $N(\pi, n-1, p)=\left(\begin{array}{c}n-1 \\ 2\end{array}\right)+1$, that is, $\left(\begin{array}{c}n-1 \\ 2\end{array}\right)=n-2$. As we have seen above, this yields a contradiction to our assumption that $n \geqslant 5$.

Suppose that $(\alpha, \beta)$ and $\left(\alpha^{\prime}, \beta^{\prime}\right)$ are of types $(\mathrm{C})$ and $(\mathrm{K})$, respectively. Then $n-1=$ $N(\pi, n-1, p)=\left(\begin{array}{c}n-2 \\ 2\end{array}\right)$, which implies $n^{2}-7 n+8=0$. The solution of this quadratic equation is $n=(7 \pm \sqrt{17}) / 2$. Since there is no integer solution, we have arrived in a contradiction.

Suppose that $(\alpha, \beta)$ and $\left(\alpha^{\prime}, \beta^{\prime}\right)$ are of types $(\mathrm{E})$ and $(\mathrm{G})$, respectively. By $C\left(\alpha^{\prime}, \beta^{\prime}\right)$ we have $q=n-1$, and by $C(\alpha, \beta)$ we have $p=q-1=n-2$. Since a condition of type (E) holds, we have $1 \leqslant p \leqslant 2$, which implies $3 \leqslant n \leqslant 4$. This contradicts our assumption that $n \geqslant 5$. A similar argument leads to a contradiction if we suppose that $(\alpha, \beta)$ is of type $(\mathrm{E})$ or $(\mathrm{G})$ and $\left(\alpha^{\prime}, \beta^{\prime}\right)$ is of type $(\mathrm{K})$.

Let us then consider the case when $|T|=3$. Assume that $T=\{p, q, r\}$ with $p<q<r$. Suppose first that $(\alpha, \beta)$ and $\left(\alpha^{\prime}, \beta^{\prime}\right)$ are of types $(\mathrm{H})$ and $(\mathrm{J})$, respectively. Condition $C(\alpha, \beta)$ implies that $q=p+1, r=q+1=p+2$, and condition $C\left(\alpha^{\prime}, \beta^{\prime}\right)$ asserts that $r=n-1$. Hence $n=r+1=p+3$. We have $n-r=N(\pi, n-1, r)=n-2$, which implies $r=2$. Hence $n=3$, which contradicts the assumption that $n \geqslant 5$.

Suppose that $(\alpha, \beta)$ is of type $(\mathrm{F})$ and $\left(\alpha^{\prime}, \beta^{\prime}\right)$ is of type $(\mathrm{H})$ or $(\mathrm{J})$. Since a condition of type $(\mathrm{F})$ holds, we have $1 \leqslant p \leqslant 2$, and since a condition of type $(\mathrm{H})$ or $(\mathrm{J})$ holds, we 
have $2 \leqslant p \leqslant n-3$. Hence $p=2$, and we have $\left(\begin{array}{c}n-1 \\ 2\end{array}\right)+1=N(\pi, n-1, p)=\left(\begin{array}{l}p \\ 2\end{array}\right)=\left(\begin{array}{l}2 \\ 2\end{array}\right)=1$, which implies that $\left(\begin{array}{c}n-1 \\ 2\end{array}\right)=0$, i.e., $n \leqslant 2$, which contradicts the assumption that $n \geqslant 5$.

Suppose that $(\alpha, \beta)$ and $\left(\alpha^{\prime}, \beta^{\prime}\right)$ are of types $(\mathrm{B})$ and $(\mathrm{F})$, respectively. Then we have $n-1=N(\pi, n-1, p)=\left(\begin{array}{c}n-1 \\ 2\end{array}\right)+1$, that is, $\left(\begin{array}{c}n-1 \\ 2\end{array}\right)=n-2$. As we have seen above, this yields a contradiction to $n \geqslant 5$.

Suppose then that $(\alpha, \beta)$ and $\left(\alpha^{\prime}, \beta^{\prime}\right)$ are of types $(\mathrm{B})$ and $(\mathrm{H})$, respectively. Condition $C\left(\alpha^{\prime}, \beta^{\prime}\right)$ implies that $p, q, r$ are three consecutive integers, i.e., $q=p+1$ and $r=p+2$. From the conditions on $N(\pi, n-1, i)$ for $i \in\{p, q, r\}$ we obtain the following system of simultaneous equations:

$$
\left\{\begin{array}{l}
\left(\begin{array}{l}
p \\
2
\end{array}\right)=n-1 \\
\left(\begin{array}{c}
n-1 \\
2
\end{array}\right)-\left(\begin{array}{l}
p \\
2
\end{array}\right)+p+1=\left(\begin{array}{c}
p+1 \\
2
\end{array}\right), \\
n-p-2=\left(\begin{array}{c}
n-1 \\
2
\end{array}\right)-\left(\begin{array}{c}
p+1 \\
2
\end{array}\right) .
\end{array}\right.
$$

Rewriting the second equation, using the identity $\left(\begin{array}{l}p \\ 2\end{array}\right)+p=\left(\begin{array}{c}p+1 \\ 2\end{array}\right)$ (see identity (1)), and then substituting $n-1$ for $\left(\begin{array}{l}p \\ 2\end{array}\right)$ as per the first equation, we obtain $\left(\begin{array}{c}n-1 \\ 2\end{array}\right)+1=2(n-1)$, which yields the quadratic equation $n^{2}-7 n+8=0$, which, as we have seen above, has no integer solution, and we have arrived in a contradiction again.

Finally, suppose that $(\alpha, \beta)$ and $\left(\alpha^{\prime}, \beta^{\prime}\right)$ are of types $(\mathrm{B})$ and $(\mathrm{J})$, respectively. By $C(\alpha, \beta)$ we have $q=r-1$, and by $C\left(\alpha^{\prime}, \beta^{\prime}\right)$ we have $p=q-1$ and $r=n-1$. Consequently, $p=n-3, q=n-2, r=n-1$. From the conditions on $N(\pi, n-1, i)$ for $i \in\{p, q, r\}$ we obtain the following system of simultaneous equations:

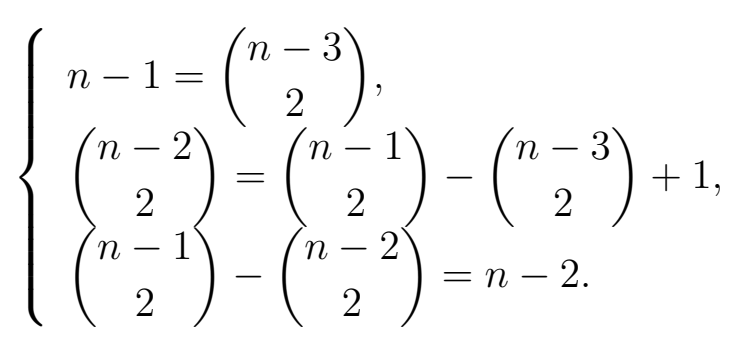

The first equation can be written equivalently as $n^{2}-9 n+14=0$. The solution of this quadratic equation is $n=2$ or $n=7$. The former solution is not feasible, because we are assuming that $n \geqslant 5$. The other two equations are also satisfied with $n=7$. Then we have $(\alpha, \beta)=(4,6)$ and $\left(\alpha^{\prime}, \beta^{\prime}\right)=(7,5)$. It is indeed true that conditions $C(4,6)$ and $C(7,5)$ are identical when $n=7$.

We conclude that conditions $C(\alpha, \beta)$ and $C\left(\alpha^{\prime}, \beta^{\prime}\right)$ hold simultaneously if and only if $(\alpha, \beta)=\left(\alpha^{\prime}, \beta^{\prime}\right)$ or $n=7$ and $\left\{(\alpha, \beta),\left(\alpha^{\prime}, \beta^{\prime}\right)\right\}=\{(4,6),(7,5)\}$. This completes the proof.

Lemma 3.5. Let $n \geqslant 5$ and $\pi \in \Sigma_{n}$. Let $k \in[n-2]$. Write $\alpha:=\pi^{-1}(k), \beta:=\pi^{-1}(k+1)$. 
For $i \in[n-1]$, let

$$
M(\pi, k, i):= \begin{cases}N(\pi, k, i), & \text { if } i \in[n] \backslash\{\beta-1, \beta\} \\ N(\pi, k, i)-\left|S_{1}^{\pi, k}\right|, & \text { if } i=\beta-1 \\ N(\pi, k, i)-\left|S_{2}^{\pi, k}\right|, & \text { if } i=\beta\end{cases}
$$

Let $m:=\max \{i \in[n-1]: M(\pi, k, i)>0\}$. Then the following statements hold:

(i) For $p \in\{1, \ldots, n-2\}, \alpha=p$ if and only if $m=p$.

(ii) $\alpha=n-1$ if and only if $m=n-1$ and $M(\pi, k, n-2) \geqslant 2$.

(iii) $\alpha=n$ if and only if $m=n-1$ and $M(\pi, k, n-2) \leqslant 1$.

Proof. We are first going to prove the forward implications of statements (i)-(iii). It follows from Lemmas 3.1 and 3.2 that $M(\pi, k, i)>0$ only if $i \leqslant \alpha$. By Lemma 3.3, $\left|S_{4}^{\pi, k}\right|=\left|S_{6}^{\pi, k}\right|=0$ if and only if $n=\alpha$. Therefore, if $\alpha<n$, then $M(\pi, k, \alpha) \geqslant$ $\left|S_{4}^{\pi, k}\right|+\left|S_{6}^{\pi, k}\right|>0$, and we have $m=\alpha$.

Furthermore, if $\alpha=n-1$, then $h_{\pi}(k) \in\{n-k-1, n-k\}$. If $h_{\pi}(k)=n-k-1$, then

$$
\begin{aligned}
M(\pi, k, n-2) \geqslant\left|S_{5}^{\pi, k}\right| & =\left(\begin{array}{c}
n-2 \\
2
\end{array}\right)-\left(\begin{array}{c}
n-2-(n-k-1) \\
2
\end{array}\right) \\
& =\left(\begin{array}{c}
n-k-1 \\
2
\end{array}\right)+(k-1)(n-k-1),
\end{aligned}
$$

where the last equality holds by identity (2). If $k=1$, then $\left(\begin{array}{c}n-k-1 \\ 2\end{array}\right)+(k-1)(n-k-1)=$ $\left(\begin{array}{c}n-2 \\ 2\end{array}\right) \geqslant\left(\begin{array}{l}3 \\ 2\end{array}\right)=3$. If $k=n-2$, then $\left(\begin{array}{c}n-k-1 \\ 2\end{array}\right)+(k-1)(n-k-1)=\left(\begin{array}{l}1 \\ 2\end{array}\right)+(n-3) \cdot 1=n-3 \geqslant 2$. If $2 \leqslant k \leqslant n-3$, then $\left(\begin{array}{c}n-k-1 \\ 2\end{array}\right)+(k-1)(n-k-1) \geqslant\left(\begin{array}{l}2 \\ 2\end{array}\right)+1 \cdot 2=3$.

If $h_{\pi}(k)=n-k$, then

$$
M(\pi, k, n-2) \geqslant\left|S_{5}^{\pi, k}\right|=\left(\begin{array}{c}
n-2 \\
2
\end{array}\right)-\left(\begin{array}{c}
n-2-(n-k) \\
2
\end{array}\right)=\left(\begin{array}{c}
n-k \\
2
\end{array}\right)+(k-2)(n-k),
$$

where the last equality holds by identity (2). In this case, we necessarily have $k>1$. If $k=2$, then $\left(\begin{array}{c}n-k \\ 2\end{array}\right)+(k-2)(n-k)=\left(\begin{array}{c}n-2 \\ 2\end{array}\right) \geqslant\left(\begin{array}{c}3 \\ 2\end{array}\right)=3$. If $3 \leqslant k \leqslant n-2$, then $\left(\begin{array}{c}n-k \\ 2\end{array}\right)+(k-2)(n-k) \geqslant\left(\begin{array}{l}2 \\ 2\end{array}\right)+1 \cdot 2=3$.

If $\alpha=n$, then $\left|S_{4}^{\pi, k}\right|=\left|S_{6}^{\pi, k}\right|=0$ and

$$
M(\pi, k, n-1) \geqslant\left|S_{5}^{\pi, k}\right|=\left|S_{5}^{\pi, k}\right|+\left|S_{6}^{\pi, k}\right|=\left(\begin{array}{c}
n-k \\
2
\end{array}\right)+(k-1)(n-k) \geqslant 1 .
$$

Moreover, we have $N(\pi, k, n-2)=\lambda+\mu$, where

$$
\lambda=\left\{\begin{array}{ll}
0, & \text { if } \pi(n-2)<k, \\
1, & \text { if } \pi(n-2)>k,
\end{array} \quad \mu= \begin{cases}0, & \text { if } n-2 \notin\{\beta-1, \beta\} \\
\left|S_{1}^{\pi, k}\right|, & \text { if } n-2=\beta-1 \\
\left|S_{2}^{\pi, k}\right|, & \text { if } n-2=\beta\end{cases}\right.
$$


Hence $M(\pi, k, n-1)=N(\pi, k, n-1)-\mu=\lambda \leqslant 1$.

We have shown above that the conditions of statements (i)-(iii) are necessary. The conditions are also sufficient, because they are clearly mutually exclusive and all possible values of $\alpha$ are covered.

Lemma 3.5 takes a simpler form when $k=1$. Namely, in this case we have $\left(\begin{array}{l}k \\ 2\end{array}\right)=0$, so $\left|S_{1}^{\pi, 1}\right|=\left|S_{2}^{\pi, 1}\right|=0$. Consequently, $M(\pi, 1, i)=N(\pi, 1, i)$ for all $i \in[n-1]$.

Corollary 3.6. Let $n \geqslant 5$ and $\pi \in \Sigma_{n}$. Let $m:=\max \{i \in[n-1]: N(\pi, 1, i)>0\}$.

(i) For $p \in\{1, \ldots, n-2\}, \pi^{-1}(1)=p$ if and only if $m=p$.

(ii) $\pi^{-1}(1)=n-1$ if and only if $m=n-1$ and $N(\pi, 1, n-2) \geqslant 2$.

(iii) $\pi^{-1}(1)=n$ if and only if $m=n-1$ and $N(\pi, 1, n-2) \leqslant 1$.

Theorem 3.7. Assume that $n \geqslant 5$, and let $\pi, \tau \in \Sigma_{n}$. Then $\operatorname{deck} \pi=\operatorname{deck} \tau$ if and only if $\pi=\tau$.

Proof. If is obvious that if $\pi=\tau$ then $\operatorname{deck} \pi=\operatorname{deck} \tau$. Assume that $\operatorname{deck} \pi=\operatorname{deck} \tau$. We are going to show by induction on $k$, starting from $k=n$ and going down to $k=1$, that $\pi^{-1}(k)=\tau^{-1}(k)$ for all $k \in[n]$. Since $\operatorname{deck} \pi=\operatorname{deck} \tau$, it clearly holds that $N(\pi, k, i)=$ $N(\tau, k, i)$ for all $k, i \in[n-1]$. The conditions of Lemma 3.4 depend only on the deck of the permutation considered, so Lemma 3.4 implies that $\pi^{-1}(n-1)=\tau^{-1}(n-1)$ and $\pi^{-1}(n)=\tau^{-1}(n)$. Let $k \in[n-2]$ and assume that $\pi^{-1}(\ell)=\tau^{-1}(\ell)$ whenever $k+1 \leqslant \ell \leqslant$ $n$. Then $h_{\pi}(k+1)=h_{\tau}(k+1)$. Consequently, $\left|S_{1}^{\pi, k}\right|=\left|S_{1}^{\tau, k}\right|$ and $\left|S_{2}^{\pi, k}\right|=\left|S_{2}^{\tau, k}\right|$, as given by Lemma 3.2, so the numbers $M(\pi, k, i)$ and $M(\tau, k, i)$ of Lemma 3.5 must be equal, for each $i \in[n-1]$. Therefore, Lemma 3.5 implies that $\pi^{-1}(k)=\tau^{-1}(k)$. This completes the inductive step, and we conclude that $\pi=\tau$.

Remark 3.8. If $n=4$, then conditions $C(2,3)$ (of type (A)) and $C(4,2)$ (of type $(\mathrm{F})$ ) of Lemma 3.4 are identical. This perhaps explains the non-reconstructibility of the permutations 1342 and 1423 (see Table 1).

\section{Permutation reconstruction algorithm}

The proof of Theorem 3.7 and Lemmas 3.4 and 3.5 provide an algorithm for recovering a permutation from its identification minors. Namely, assume that we are given as an input $\operatorname{deck} \pi$ for some permutation $\pi \in \Sigma_{n}, n \geqslant 5$, but the permutation $\pi$ itself is unknown. We can then apply the following procedure to determine $\pi$.

1. Determine the numbers $N(\pi, k, i)$ for all $k \in[n], i \in[n-1]$. (This is straightforward calculation from the input.)

2. By examining the numbers $N(\pi, n-1, i)$, determine the unique elements $\alpha, \beta \in[n]$ for which condition $C(\alpha, \beta)$ of Lemma 3.4 holds. Unless we have $n=7$ and $(\alpha, \beta) \in$ $\{(4,6),(7,5)\}$, we can set $\pi^{-1}(n-1):=\alpha$ and $\pi^{-1}(n):=\beta$; otherwise we need to check the special additional conditions of Lemma 3.4 (ii) and (iii) to determine 


\begin{tabular}{rr|rrrrrr}
\multicolumn{7}{r|}{$N(\pi, k, i)$} & \multicolumn{6}{|c}{$i$} \\
& 1 & 2 & 3 & \multicolumn{1}{c}{4} & \multicolumn{1}{c}{5} & \multicolumn{1}{c}{6} \\
\hline \multirow{4}{*}{$k$} & 1 & 1 & 1 & 1 & 1 & 11 & 6 \\
& 2 & 1 & 2 & 4 & 14 & 0 & 0 \\
& 3 & 1 & 17 & 0 & 0 & 0 & 3 \\
& 4 & 1 & 0 & 1 & 1 & 6 & 12 \\
& 5 & 1 & 0 & 11 & 5 & 4 & 0 \\
& 6 & 16 & 1 & 4 & 0 & 0 & 0
\end{tabular}

Table 5: The numbers $N(\pi, k, i)$ of Example 4.1.

$\pi^{-1}(n)$ and $\pi^{-1}(n-1)$. (This can be done by examining directly the cards that are given as an input.)

3. For each $k$, starting from $n-2$ and going down to 1, perform the following: Compute the numbers $M(\pi, k, i)(i \in[n-1])$ of Lemma 3.5 and determine $\pi^{-1}(k)$ by applying Lemma 3.5. (Observe that the numbers $M(\pi, k, i)$ can indeed be computed, because we know $N(\pi, k, i)$ for all $k, i \in[n-1]$ and we have already determined $\pi^{-1}(\ell)$ for $\ell>k$. In particular we know $\pi^{-1}(k+1)$ and the number $h_{\pi}(k+1)$, which occurs in the formula for $\left|S_{1}^{\pi, k}\right|$ and $\left|S_{2}^{\pi, k}\right|$ in Lemma 3.2.)

Some shortcuts can be made in this algorithm. By Corollary 3.6 and Lemma 3.5, we can actually determine $\pi^{-1}(1)$ from the numbers $N(\pi, 1, i)(i \in[n-1])$ without first determining $\pi^{-1}(\ell)$ for any other $\ell \in[n]$. Note also that if $I=\{n, \ell\}$, where $\ell$ is an element of $[n]$ such that $\pi^{-1}(\ell) \in\left\{\pi^{-1}(n)-1, \pi^{-1}(n)+1\right\}$, then the string $\pi^{I}(1) \pi^{I}(2) \ldots \pi^{I}(n-1)$ is obtained from $\pi(1) \pi(2) \ldots \pi(n)$ simply by removing the entry $n=\pi\left(\pi^{-1}(n)\right)$. Thus, we could proceed as follows. First determine $\pi^{-1}(1), \pi^{-1}(n-1)$, and $\pi^{-1}(n)$ using Lemmas 3.4 and 3.5. Then we can start determining the entries as above, going down from $\ell=n-2$ to $\ell=2$, but we can stop the iteration at any point when there is only one card, possibly with multiplicity greater than 1 , where the entries determined so far are in the right positions (taking into account the deletion of $n$, whose position is known). By inserting $n$ at the appropriate position in this string, we obtain $\pi$.

Let us illustrate the permutation reconstruction algorithm with an example.

Example 4.1. Assume that $n=7$ and $\pi \in \Sigma_{n}$ is a permutation such that

$$
\begin{aligned}
\operatorname{deck} \pi=\{ & 136254,236514,362514,436251,536214,615243,625143,625413,631254, \\
& 632514,632514,632514,634251,635214,635214,635214,635214,635241, \\
& 635241,635241,635241\} .
\end{aligned}
$$

In order to determine $\pi$, let us first count how many times each number appears at each position in the cards of $\pi$. The values $N(\pi, k, i)$ for each $k \in[n-1], i \in[n-1]$ are shown in Table 5. Based on this information, we can determine $\pi^{-1}(6)$ and $\pi^{-1}(7)$ by applying Lemma 3.4. We see that condition $C(3,1)$ of type $(\mathrm{F})$ holds: we have $T=\{i \in$ [6] : $N(\pi, 6, i)>0\}=\{1,2,3\}, N(\pi, 6,1)=\left(\begin{array}{c}7-1 \\ 2\end{array}\right)+1=16, N(\pi, 6,2)=2-1=1$, 


\begin{tabular}{ccccccccccccc}
\multicolumn{10}{c}{} & \multicolumn{10}{c}{$M(\pi, k, i)$} \\
$k$ & $h_{\pi}(k+1)$ & $\left|S_{1}^{\pi, k}\right|$ & $\left|S_{2}^{\pi, k}\right|$ & $i:$ & 1 & 2 & 3 & 4 & 5 & 6 & $\pi^{-1}(k)$ & $\pi$ \\
\hline 5 & 1 & $\left(\begin{array}{c}3-1-1 \\
2\end{array}\right)=0$ & $\left(\begin{array}{c}5 \\
2\end{array}\right)-0=10$ & 1 & 0 & 1 & 5 & 4 & 0 & 5 & $7 * 6 * 5 * *$ \\
4 & 2 & $\left(\begin{array}{c}5-1-2 \\
2\end{array}\right)=1$ & $\left(\begin{array}{l}4 \\
2\end{array}\right)-1=5$ & 1 & 0 & 1 & 0 & 1 & 12 & 7 & $7 * 6 * 5 * 4$ \\
3 & 3 & $\left(\begin{array}{c}7-1-3 \\
2\end{array}\right)=3$ & $\left(\begin{array}{l}3 \\
2\end{array}\right)-3=0$ & 1 & 17 & 0 & 0 & 0 & 0 & 2 & $736 * 5 * 4$ \\
2 & 1 & $\left(\begin{array}{c}2-1-1 \\
2\end{array}\right)=0$ & $\left(\begin{array}{l}2 \\
2 \\
2\end{array}\right)-0=1$ & 1 & 1 & 4 & 14 & 0 & 0 & 4 & $73625 * 4$ \\
1 & 3 & $\left(\begin{array}{c}4-1-3 \\
2\end{array}\right)=0$ & $\left(\begin{array}{l}1 \\
2\end{array}\right)-0=0$ & 1 & 1 & 1 & 1 & 11 & 6 & 6 & 7362514
\end{tabular}

Table 6: Data generated by the reconstruction algorithm in Example 4.1.

$N(\pi, 6,3)=7-3=4$. Therefore $\pi^{-1}(6)=3, \pi^{-1}(7)=1$, so $\pi=7 * 6 * * * *$, where the $*$ 's denote values of $\pi$ that are still unknown to us.

Now, applying Lemmas 3.2 and 3.5, we can determine the data shown in Table 6, filling in the cells in the usual reading order: from left to right and from top to bottom. We conclude that $\pi=7362514$.

Note that we could make a shortcut. We can determine $\pi^{-1}(6)=3, \pi^{-1}(7)=1$, $\pi^{-1}(1)=6$ as above, so we know that $\pi=7 * 6 * * 1 *$. Hence there must be a card of the form $* 6 * * 1 *$. In fact, there is only one card of this form, namely 362514 . Inserting 7 in the first position we recover $\pi=7362514$.

\section{Set-reconstructibility of permutations}

Having discovered that all permutations of $[n]$ are reconstructible from identification minors whenever $n \geqslant 5$, we may ask whether reconstruction is possible if we ignore the multiplicities of cards. As we will see shortly, this is not in general possible, even for large values of $n$.

The set-deck of a permutation $\pi \in \Sigma_{n}$ is defined as the set $\left\{\pi^{I}: I \in\left(\begin{array}{c}{[n]} \\ 2\end{array}\right)\right\}$ of its identification minors, and it is denoted by set-deck $\pi$. If $\tau \in \Sigma_{n}$ and set-deck $\pi=\operatorname{set}-\operatorname{deck} \tau$, then $\tau$ is a set-reconstruction of $\pi$. If every set-reconstruction of $\pi$ is equal to $\pi$, then $\pi$ is set-reconstructible. Set-reconstructibility clearly implies reconstructibility, but the converse is not necessarily true.

If $2 \leqslant n \leqslant 4$, then there obviously exist permutations of $[n]$ that are not set-reconstructible, for the simple reason that there are permutations that are not even reconstructible. Further examples of permutations that are not set-reconstructible, despite being reconstructible, can be found by examining Table 1 .

By Theorem 3.7, every permutation of a set with at least five elements is reconstructible, but, as the following example illustrates, for every $n \geqslant 3$, there exist non-setreconstructible permutations of $[n]$.

Example 5.1. Assume that $n \geqslant 3$, and let $\pi=23 \ldots n 1, \tau=23 \ldots(n-1) 1 n$. Let us consider the identification minors of $\pi$ and $\tau$. Let $I=\{i, j\} \in\left(\begin{array}{c}{[n]} \\ 2\end{array}\right)$ with $i<j$. If $i=1$, 


\begin{tabular}{|c|c|c|c|c|c|}
\hline$\Pi$ & $\pi^{\Pi}$ & $\Pi$ & $\pi^{\Pi}$ & $\Pi$ & $\pi^{\Pi}$ \\
\hline$\{\{1,2,3\},\{4\},\{5\}\}$ & 132 & $\{\{3,4,5\},\{1\},\{2\}\}$ & 312 & $\{\{1,4\},\{3,5\},\{2\}\}$ & 312 \\
\hline$\{\{1,2,4\},\{3\},\{5\}\}$ & 213 & $\{\{1,2\},\{3,4\},\{5\}\}$ & 213 & $\{\{1,5\},\{2,3\},\{4\}\}$ & 213 \\
\hline$\{\{1,2,5\},\{3\},\{4\}\}$ & 213 & $\{\{1,2\},\{3,5\},\{4\}\}$ & 213 & $\{\{1,5\},\{2,4\},\{3\}\}$ & 312 \\
\hline$\{\{1,3,4\},\{2\},\{5\}\}$ & 132 & $\{\{1,2\},\{4,5\},\{3\}\}$ & 213 & $\{\{1,5\},\{3,4\},\{2\}\}$ & 312 \\
\hline$\{\{1,3,5\},\{2\},\{4\}\}$ & 123 & $\{\{1,3\},\{2,4\},\{5\}\}$ & 132 & $\{\{2,3\},\{4,5\},\{1\}\}$ & 213 \\
\hline$\{\{1,4,5\},\{2\},\{3\}\}$ & 312 & $\{\{1,3\},\{2,5\},\{4\}\}$ & 123 & $\{\{2,4\},\{3,5\},\{1\}\}$ & 312 \\
\hline$\{\{2,3,4\},\{1\},\{5\}\}$ & 213 & $\{\{1,3\},\{4,5\},\{2\}\}$ & 132 & $\{\{2,5\},\{3,4\},\{1\}\}$ & 312 \\
\hline$\{\{2,3,5\},\{1\},\{4\}\}$ & 213 & $\{\{1,4\},\{2,3\},\{5\}\}$ & 213 & & \\
\hline$\{\{2,4,5\},\{1\},\{3\}\}$ & 312 & $\{\{1,4\},\{2,5\},\{3\}\}$ & 312 & & \\
\hline
\end{tabular}

Table 7: Permutations arising from $\pi=31524$ via 3-partitions.

then $\pi_{I}=\tau_{I}=2 \ldots(j-1) 1 j \ldots(n-1)$. If $i \geqslant 2$, then $\pi_{I}=23 \ldots(n-1) 1$. If $i \geqslant 2$ and $j \leqslant n-1$, then $\tau_{I}=23 \ldots(n-2) 1(n-1)$. If $i \geqslant 2$ and $j=n$, then $\tau_{I}=23 \ldots(n-1) 1$. We conclude that $\pi$ and $\tau$ have the same set-deck, namely

$$
\{123 \ldots(n-1), 213 \ldots(n-1), 2314 \ldots(n-1), \ldots, 23 \ldots(n-1) 1\} .
$$

\section{Generalization}

The identification minors of permutations that we have discussed in this paper are a special case of a more general way of deriving permutations from a given permutation that was studied by the current author in [10]. Namely, given a permutation $\pi=\pi_{1} \pi_{2} \ldots \pi_{n} \in \Sigma_{n}$ and a partition $\Pi$ of $[n]$ with $\ell$ blocks, we define the permutation $\pi^{\Pi}$ of $[\ell]$ as the result of the following procedure:

1. For each block $B$ of $\Pi$, let $m_{B}:=\min \left\{\pi_{i}: i \in B\right\}$, and for every $i \in B$, replace $\pi_{i}$ by $m_{B}$.

2. Remove all repeated occurrences of elements from the resulting sequence, retaining only the first occurrence of each number $m_{B}(B \in \Pi)$.

3. Take the pattern of the resulting sequence.

We say that $\pi^{\Pi}$ is the permutation arising from $\pi$ via $\Pi$. For $k \in \mathbb{N}_{+}$, let us call $\pi^{\Pi}$ an $(n-k)$-identification minor of $\pi$ if $\Pi$ has $n-k$ blocks. For example, the permutations arising from $\pi=31524$ via 3-partitions are presented in Table 7. Using this terminology, the identification minors of $\pi$ that we have discussed earlier in this paper are $(n-1)$ identification minors, as they are exactly the permutations arising from $\pi$ via partitions of $[n]$ with $n-1$ blocks, i.e., partitions of the form $\{I\} \cup\{\{i\}: i \in[n] \backslash I\}$ for some $I \in\left(\begin{array}{c}{[n]} \\ 2\end{array}\right)$.

This definition gives immediately rise to a reconstruction problem: for a fixed parameter $k \in \mathbb{N}_{+}$, the objects are permutations of an $n$-element set, the cards of a permutation 
$\pi \in \Sigma_{n}$ are its $(n-k)$-identification minors $\pi^{\Pi}$ for each $(n-k)$-partition $\Pi$ of $[n]$, and the equivalence relation on $\Sigma_{n}$ is the equality relation.

Investigation of this reconstruction problem for $k>1$ remains a topic of future research. For example, the following problem seems natural. For $k \in \mathbb{N}_{+}$, let us denote by $N_{k}$ the smallest number $N$, if one exists, such that for every $n \geqslant N$, every permutation of $[n]$ is reconstructible from its $(n-k)$-identification minors. Theorem 3.7 and the discussion in Section 2 show that $N_{1}=5$. Does the number $N_{k}$ exist for every positive integer $k$ ? Determine $N_{k}$ for $k>1$.

\section{Acknowledgments}

The author would like to thank the anonymous referee for his or her valuable suggestions and comments that helped improve the quality of the manuscript.

The work reported in this paper was carried out while the author was working at the University of Lisbon. The author is currently affiliated with TU Dresden. This work was developed within the FCT project CEMAT-CIÊNCIAS (UID/Multi/04621/2013).

\section{References}

[1] M. Couceiro and S. Foldes. On closed sets of relational constraints and classes of functions closed under variable substitutions. Algebra Universalis 54:149-165, 2005.

[2] M. Couceiro, E. Lehtonen, and K. Schölzel. A complete classification of equational classes of threshold functions included in clones. RAIRO Oper. Res. 49:39-66, 2015.

[3] M. Couceiro, E. Lehtonen, and K. Schölzel. Set-reconstructibility of Post classes. Discrete Appl. Math. 187:12-18, 2015.

[4] M. Couceiro, E. Lehtonen, and K. Schölzel. Hypomorphic Sperner systems and nonreconstructible functions. Order 32:255-292, 2015.

[5] O. Ekin, S. Foldes, P. L. Hammer, and L. Hellerstein. Equational characterizations of Boolean function classes. Discrete Math. 211:27-51, 2000.

[6] J. Ginsburg. Determining a permutation from its set of reductions. Ars Combin. 82:55-67, 2007.

[7] P. J. Kelly. A congruence theorem for trees. Pacific J. Math. 7:961-968, 1957.

[8] E. Lehtonen. Totally symmetric functions are reconstructible from identification minors. Electron. J. Combin. 21(2):\#P2.6, 2014.

[9] E. Lehtonen. Reconstructing multisets over commutative groupoids and affine functions over nonassociative semirings. Internat. J. Algebra Comput. 24:11-31, 2014.

[10] E. Lehtonen. Order of first occurrence. Submitted.

[11] M. Monks. Reconstructing permutations from cycle minors. Electron. J. Combin. 16(1):\#R19, 2009. 
[12] N. Pippenger. Galois theory for minors of finite functions. Discrete Math. 254:405419, 2002.

[13] M. Raykova. Permutation reconstruction from minors. Electron. J. Combin. 13:\#R66, 2006.

[14] R. Smith. Permutation reconstruction. Electron. J. Combin. 13:\#N11, 2006.

[15] S. M. Ulam. A Collection of Mathematical Problems. Interscience Tracts in Pure and Applied Mathematics, no. 8. Interscience Publishers, New York-London, 1960.

[16] C. Wang. Boolean minors. Discrete Math. 141:237-258, 1995.

[17] R. Willard. Essential arities of term operations in finite algebras. Discrete Math. 149:239-259, 1996.

[18] I. E. Zverovich. Characterization of closed classes of Boolean functions in terms of forbidden subfunctions and Post classes. Discrete Appl. Math. 149:200-218, 2005. 